\title{
Hepatic fibrosis and factors associated with liver stiffness in HIV mono-infected individuals
}

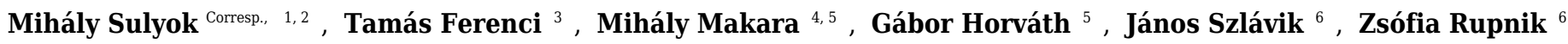 \\ , Luca Kormos ${ }^{6}$, Zsuzsanna Gerlei ${ }^{7}$, Zita Sulyok ${ }^{2}$, István Vályi-Nagy ${ }^{4,6}$ \\ 1 Doctoral School for Clinical Medicine, Semmelweis University, Budapest, Hungary \\ 2 Institute for Tropical Medicine, Eberhard Karls University, Tuebingen, Germany \\ 3 John von Neumann Faculty of Informatics, Physiological Controls Group, Óbuda University, Budapest, Hungary \\ 4 Center for Hepatology, St. István and St László Hospital, Budapest, Hungary \\ 5 Hepatology Center of Buda, Budapest, Hungary \\ 6 Center for HIV, St. István and St László Hospital, Budapest, Hungary \\ 7 Transplantation and Surgical Clinic, Semmelweis University, Budapest, Hungary \\ Corresponding Author: Mihály Sulyok \\ Email address: mihaly.sulyok@uni-tuebingen.de
}

Background. Liver disease has become an important cause of morbidity and mortality even in those HIV-infected individuals who are devoid of hepatitis virus co-infection. The aim of this study was to evaluate the degree of hepatic fibrosis and the role of associated factors using liver stiffness measurement in HIV mono-infected patients without significant alcohol intake. Methods. We performed a cross-sectional study of 101 HIV mono-infected patients recruited prospectively from March 1, 2014 to October 30, 2014 at the Center for HIV, St István and St László Hospital, Budapest, Hungary. To determine hepatic fibrosis, liver stiffness was measured with transient elastography. Demographic, immunologic and other clinical parameters were collected to establish a multivariate model. Bayesian Model Averaging (BMA) was performed to identify predictors of liver stiffness. Results. Liver stiffness ranged from 3.0-34.3 kPa, with a median value of $5.1 \mathrm{kPa}$ (IQR 1.7). BMA provided a very high support for age (Posterior Effect Probability-PEP: 84.5\%), moderate for BMI (PEP: 49.3\%), CD4/8 ratio (PEP: 44.2\%) and lipodystrophy (PEP: 44.0\%). For all remaining variables, the model rather provides evidence against their effect. These results overall suggest that age and BMI have a positive association with LS, while CD4/8 ratio and lipodystrophy are negatively associated. Discussion. Our findings shed light on the possible importance of ageing, overweight and HIV-induced immune dysregulation in the development of liver fibrosis in the HIV-infected population. Nonetheless, further controlled studies are warranted to clarify causal relations. 
1 Hepatic fibrosis and factors associated with liver stiffness in HIV mono2 infected individuals

4 Mihály Sulyok ${ }^{1,2}$, Tamás Ferenci ${ }^{3}$, Mihály Makara ${ }^{4}$, Gábor Horváth ${ }^{5}$, János Szlávik ${ }^{6}$, Zsófia

5 Rupnik $^{6}$, Luca Kormos ${ }^{6}$, Zsuzsanna Gerlei ${ }^{7}$, Zita Sulyok ${ }^{1}$, István Vályi-Nagy4, 6

$6{ }^{1}$ Doctoral School for Clinical Medicine, Semmelweis University, Budapest, Hungary

$7 \quad{ }^{2}$ Institute for Tropical Medicine, Eberhard Karls University, Tuebingen, Germany

$8{ }^{3}$ John von Neumann Faculty of Informatics, Physiological Controls Group, Óbuda University, 9 Budapest, Hungary

${ }^{4}$ Center for Hepatology, St. István and St László Hospital, Budapest, Hungary

${ }^{5}$ Hepatology Center of Buda, Budapest, Hungary

${ }^{6}$ Center for HIV, St. István and St László Hospital, Budapest, Hungary

${ }^{7}$ Transplantation and Surgical Clinic, Semmelweis University, Budapest, Hungary

Corresponding author: Mihály Sulyok 


\section{Abstract}

Background. Liver disease has become an important cause of morbidity and mortality even in those HIV-infected individuals who are devoid of hepatitis virus co-infection. The aim of this study was to evaluate the degree of hepatic fibrosis and the role of associated factors using liver stiffness measurement in HIV mono-infected patients without significant alcohol intake.

Methods. We performed a cross-sectional study of 101 HIV mono-infected patients recruited prospectively from March 1, 2014 to October 30, 2014 at the Center for HIV, St István and St László Hospital, Budapest, Hungary. To determine hepatic fibrosis, liver stiffness was measured with transient elastography. Demographic, immunologic and other clinical parameters were collected to establish a multivariate model. Bayesian Model Averaging (BMA) was performed to identify predictors of liver stiffness.

Results. Liver stiffness ranged from $3.0-34.3 \mathrm{kPa}$, with a median value of $5.1 \mathrm{kPa}$ (IQR 1.7). BMA provided a very high support for age (Posterior Effect Probability-PEP: 84.5\%), moderate for BMI (PEP: 49.3\%), CD4/8 ratio (PEP: 44.2\%) and lipodystrophy (PEP: 44.0\%). For all remaining variables, the model rather provides evidence against their effect. These results overall suggest that age and BMI have a positive association with LS, while CD4/8 ratio and lipodystrophy are negatively associated.

Discussion. Our findings shed light on the possible importance of ageing, overweight and HIVinduced immune dysregulation in the development of liver fibrosis in the HIV-infected population. Nonetheless, further controlled studies are warranted to clarify causal relations. 


\section{Introduction}

Liver disease has become one of the most important cause of morbidity and mortality in HIV-infected individuals (Weber et al. 2006). While hepatitis B or C co-infections remain the most important cause of liver damage, liver related mortality also affects those infected only with HIV (Antiretroviral Therapy Cohort Collaboration 2010). Long term antiretroviral and nonantiretroviral medications, HIV induced long term inflammation, metabolic complications and direct cytopathic effects may also contribute to the pathogenesis of liver fibrosis (LF) (Rockstroh et al. 2014). An increasing number of papers have been published on fibrosis in HIV/hepatitis virus co-infected patients (Audsley et al. 2016; Brunet et al. 2016; Costiniuk et al. 2016; Fernández-Montero et al. 2013; Gonzalez et al. 2015; Ioannou et al. 2015; Kliemann et al. 2016; Konerman et al. 2014; Kooij et al. 2016; Li Vecchi et al. 2013; Macías et al. 2013a; Macías et al. 2013b; Njei et al. 2016; Sanmartín et al. 2014; Vergara et al. 2007) but only a few studies have appeared on the analysis of data obtained from HIV mono-infected individuals (Akhtar et al. 2008; DallaPiazza et al. 2010; Han et al. 2013; Lui et al. 2016; Rivero-Juárez et al. 2013; Shur et al. 2016). index (APRI) and the FIB-4 score, cross-sectional and prospective studies to evaluate prevalence and incidence of LF in HIV-infected individuals have become easier. These tests were demonstrated to be acceptable in predicting the absence of fibrosis or mild fibrosis ( $\mathrm{LF}<2$ 
71 METAVIR score) and the presence of advanced fibrosis (LF > 3 METAVIR score) (González

72 Guilabert et al. 2010). Cross-sectional studies in HIV mono-infected patients reported high rates

73 (11-47\%) of significant LF suggesting that HIV itself may contribute independently to liver

74 damage (Rockstroh et al. 2014). Ongoing LF is not always accompanied by elevated liver

75 enzymes. Thus, the diagnosis of LF and the prevention of progression to liver cirrhosis are

76 important challenges. As a result, adequate monitoring strategies of liver disease are clearly

77 needed to optimize care of HIV-infected individuals.

78

79

80

81

82

To date, only a few studies using LS measurements have examined the prevalence and potential risk factors for hepatic fibrosis among HIV mono-infected patients. Using different cutoff values resulted in a wide range in prevalence estimates (Han et al. 2013; Merchante et al. 2010). Pre-defined cutoffs adopted from the HIV/HCV-co-infected population may lead to an underestimation of the number of HIV mono-infected patients with clinically significant fibrosis as these cutoffs were determined for a population in which ongoing fibrosis is triggered by HCV co-infection (Han et al. 2013). To overcome this limitation, our aim was to use a continuous scale of LS values without any cutoff to identify significant predictors of LS in a cross-sectional study.

\section{Materials \& Methods}

\section{Study population}

The investigation was performed in accordance with the Helsinki Declaration and was approved by the Institutional Review Board of St. István and St. László Hospital, Budapest, 
94 Hungary (approval number: 34/EB/2013). Written informed consent was taken from all study

95 participants. The present cross-sectional study is an analysis of data collected for a previous

96 study, with methodology already described (Sulyok et al. 2015). Individuals older than 18 years

97 of age were enrolled after providing their written informed consent. Pregnant women and

98 patients with unreliable transient elastography measurement were excluded. Patients with known

$99 \mathrm{HCV}$ or HBV infection or anti-HBc positivity, known other risk factors of liver diseases, or

100 significant daily alcohol intake ( $>50 \mathrm{~g} /$ day) were excluded from the analysis.

101 From March 1, 2014 to October 30, 2014 all HIV-infected patients who attended the

102 outpatient clinic at the HIV Center, St. István and St. László Hospital (Budapest, Hungary) were

103 invited to participate in the study $(n=756)$. Liver stiffness measurements were performed on 139

104 patients. Out of this cohort 101 individuals were eligible for the final analysis (Fig. 1). The mode

105 of transmission of HIV was reported to be sexual intercourse in all patients. The baseline study

106 population characteristics are summarized in Table 1.

\section{Transient elastography}

Hepatology Center of Buda, Budapest, Hungary, using a FibroScan 502 equipment (Fibroscan,

111 EchoSens ${ }^{\mathrm{TM}}$, Paris, France). Measurements were performed using M probe on the right lobe of

112 the liver, through intercostal spaces according to instructions by the manufacturer. Examinations

113 with 10 successful shots and an interquartile range (IQR) for LSs less than $30 \%$ of the median

114 value were considered as reliable. Details of the technical background and the examination

115 procedure have been previously described elsewhere (Sandrin et al. 2003). We used a continuous 
116 scale of LS values in our statistical analyses to avoid information loss emerging from

117 categorization of the variable. However, to describe the patient population we adopted the cutoff

118 for significant LF of $7.2 \mathrm{kPa}$ and $5.3 \mathrm{kPa}$, and $14.6 \mathrm{kPa}$ to define the presence of cirrhosis (Han

119 et al. 2013; Vergara et al. 2007).

\section{Interview and clinical assessment}

Clinical parameters were collected on the day of transient elastography examination.

Recorded data were as follows: age, sex, body mass index (BMI), facial lipodystrophy assessment (defined by the presence deeper cheek atrophy), smoking, alcohol intake, drug use, type of antiretroviral medication (ARV), co-medications, comorbidities, and date of HIV diagnosis. Biochemical and immunological parameters, blood count, CD4 and CD8 count were collected at the visit when the informed consent was obtained $(<4$ weeks before the LS measurement).

\section{Statistical analysis}

The primary outcome variable was liver stiffness. The univariate association with categorical variables was assessed by a two independent sample Mann-Whitney $U$ test (i.e.

Wilcoxon rank-sum test). The univariate correlation with continuous variables was assessed using the Pearson and Kendall- $\tau$ rank-correlation coefficient. Visualization was performed with scattergrams indicating best fitting linear curve and LOWESS-smoother. Holm correction was performed to counteract problems related to multiple comparisons. 
139 deviation of the posterior distribution for each covariate (Hoeting et al. 1999; Raftery 1995). Best

140 models are illustrated visually by depicting the variables included in them.

141 Calculations were performed using R (R Core Team 2016) with library BMA (Raftery et

142 al. 2015). Data and script are available as supplemental information (SI and SI2).

\section{Results}

LS ranged from $3.0 \mathrm{kPa}$ to $34.3 \mathrm{kPa}$ with a median value of $5.1 \mathrm{kPa}$ (IQR 1.7). According

to the HIV/HCV co-infection LS cutoffs, significant LF defined as LS $>7.2 \mathrm{kPa}$ was detectable in 10/101 (9.9\%) individuals. Presence of cirrhosis (LS >14.6 kPa) was observed in $2(1.98 \%)$

participants. Applying the cutoff $(5.3 \mathrm{kPa})$ from a healthy population, significant fibrosis was detected in 45/101 (44.55\%) patients. attenuation parameter (CAP) value $(p=0.022985 ; p=0.0000162)$, age $(p=0.003794 ; p=0.006593)$ and BMI ( $p=0.010303 ; p=0.000146)$. With regard to categorical variables, significant association could be identified with hypertension $(p=0.04548)$ but not with ARVs. After correction due to multiple testing, only association with LS and BMI ( $p=0.0048114)$ and LS and CAP $(p=0.0005496)$ remained significant. Associations of LS and different continuous and categorical variables are presented in Tables 2-3 and Fig. 2A-I. 
$16044.0 \%$ ). On the other hand, for all remaining variables, the model rather provided evidence

161 against their effect. Figure 3 shows the best models graphically. These results overall suggest

162 that age and BMI have a positive association with LS, while CD4/8 ratio and lipodystrophy are

163 negatively associated.

It is worth noting that even the best model has only $2.4 \%$ posterior probability (even the cumulative posterior probability for the 10 best models is only $15.6 \%$ ). The best model includes age $(\beta=0.10[0.039-0.16], p=0.00174)$ and $C D 4 / 8$ ratio $(\beta=-2.2[-4.1--0.28], p=0.02501)$, but these results should be interpreted with caution in the light of the substantial model uncertainty.

\section{Discussion}

To our knowledge, only a few studies assessing liver stiffness in HIV-infected patients without $\mathrm{HBV}$ or $\mathrm{HCV}$ infection have been published so far. In these publications a wide range of prevalence for abnormal LS values were identified.(Han et al. 2013; Lui et al. 2016; Merchante et al. 2010; Rockstroh et al. 2014). Using these applied cutoff values we had a similarly wide prevalence range (9.9-44.55\%). These diverse results clearly underline the importance of identifying better cutoff values in HIV mono-infected patients. The most reliable method for this would be to perform liver biopsy in a large unselected HIV mono-infected population and to compare its results with those of transient elastography. Nevertheless, to our knowledge, no such study has been carried out. The discrepancies in cutoff values might lead to unreliable estimation of the rate and grade of LF. Therefore, we used a continuous scale of LS for our correlation and regression analyses to avoid uncertainty arising from using a pre-defined abnormal values as a cutoff point. 
BMA revealed age as the most important predictor of LS. Age is a well-known risk factor

184 for LF in non-alcoholic fatty liver disease (NAFLD) and HCV-infected patients (Chan et al.

185 2016). However, data about age-related fibrosis in the HIV mono-infected population are scarce

186 (Rockstroh et al. 2014). To date, only a few descriptive studies identified significant association

187 with age and LF in this patient population (Blanco et al. 2011; Han et al. 2013; Merchante et al.

188 2010). Ageing has multiple effects on the liver, making it more vulnerable to fibrogenetic

189 factors. The exact mechanism, however, remains unknown. The decreased regenerative capacity,

190 microbial translocation and HIV-induced immunologic dysfunction as well as chronic

191 inflammation may play non-mutually exclusive roles. (Chan et al. 2016). This result was also in

192 line with our other finding, the identified remarkable negative association between LS and

193 CD4/8 ratio. The low CD4/8 ratio is an accepted marker of HIV-induced immune dysregulation

194 (Serrano-Villar et al. 2014). Therefore, this observation could reflect on the role of HIV-induced

195 immune dysregulation in the development LF. In this population, persisting abnormally low

196 CD4/8 ratio is associated with impaired gut mucosal immunity (Serrano-Villar et al. 2014).

197 Destruction of the mucosal barrier leading to microbial translocation could be a driving force of

198 LF. In a recent study, a marker of microbial translocation, elevated sCD14 levels were associated

199 with increased LS in HIV mono-infected individuals (Redd et al. 2013). In HCV infected

200 patients, the CD4/8 ratio as a contributing factor to LF has also been considered (Feuth et al.

201 2014). Furthermore, CD4 cells can stimulate anti-fibrotic natural killer cell activity, therefore,

202 loss and impaired activity of CD4 cells may contribute to the progression of LF (Rockstroh et al.

203 2014). Data suggesting HIV-induced effects on the pathogenesis of fibrosis generation has been

204 described mainly in patients with HIV/HCV co-infection (Rockstroh et al. 2014) but the

205 mechanism has still not been exactly determined. In context of the ageing HIV population, a 
206 better understanding of how ageing interacts with HIV-induced immunologic and metabolic

207 changes will have paramount importance in reducing the burden of liver diseases.(Chan et al. 208 2016)

209 CAP value, quantifying hepatic steatosis showed significant correlation with LS in the 210 univariate analysis. Remarkably, NAFLD is the most frequent cause of liver damage in this

211 population (Rivero-Juárez et al. 2013). However, other studies found no association with LS and 212 CAP (Macías et al. 2014; Macías et al. 2016). Nonetheless, multivariate analyses rather provided 213 evidence against the effect of CAP on LS.

The correlation between BMI and LS portrays a similar profile. BMI, the most important predictor of CAP value in the HIV-infected population (Macías et al. 2014; Macías et al. 2016; association remained considerable according to the result of the BMA. This suggests, that obesity may have an independent unfavorable effect on LF even in the absence of -with CAP detectable- hepatic steatosis. the importance of antiretroviral treatment, however, other studies have raised questions about the role of older ARVs in LF development. A cumulative exposure to boosted protease inhibitors (PI) was identified as a significant independent negative predictor of LF (Han et al. 2013). A possible explanation of this result could be, that a longer cumulative boosted PI exposure may

227 reflect on a better long-term control of viral load and a lower grade of immune dysregulation.

228 Since body-fat composition abnormalities are associated with PI exposure (Grinspoon \& Carr 
229 2005), the identified negative association with the presence of facial lipodystrophy in our study

230 may further support this theory. However, prospective, controlled trials are clearly warranted to

231 clarify the role of PI therapy in the development of LF. Associations with didanosine and

232 stavudine with hepatic fibrosis were previously described (Akhtar et al. 2008; Blanco et al. 2011;

233 Merchante et al. 2010). In our investigated population the number of dideoxynucleoside exposed

234 patients was negligible $(n=2)$, therefore, we did not include these ARVs in our analysis.

235 The observed outlier value in one participant ( $\mathrm{LS}=34.3 \mathrm{kPa}$ ) refers to an advanced liver

236 disease of unknown origin. Similarly, other observational studies in the HIV mono-infected

237 population also identified individuals with high grade fibrosis and even with cryptogenic

238 cirrhosis (Lui et al. 2016; Merchante et al. 2010). Recently, cirrhosis was identified in 5.2\%

239 percent of the HIV mono-infected patients (defined as LS $>10.3 \mathrm{kPa}$ ) compared to the $0.6 \%$ of

240 the uninfected control group (Lui et al. 2016). These data underscore the importance of

241 identifying other underlying liver diseases and improving the understanding of pathomechanism.

242 It is worth contrasting these result with those obtained using traditional linear regression

243 (without variable selection). At 5\%, age $(\mathrm{p}=0.0415), \mathrm{BMI}(\mathrm{p}=0.0204)$, presence of lipodystrophy

$244(p=0.0131)$, history of taking zidovudine $(p=0.0442)$ and lopinavir $(p=0.0173)$ were significant.

245 However while this model has an apparent $\mathrm{R}^{2}$ of $36 \%$, its realistic - overfitting-optimism

246 corrected $-\mathrm{R}^{2}$ is practically zero (obtained through bootstrap validation). Thus, regularization

247 was applied - with the penalty parameter selected by Hurvich and Tsai's corrected AIC - which

248 resulted in a realistic model, however, it had no significant variable at all (Harrell 2016). This

249 experiment clearly illustrates the problems of modelling with so limited sample size, and the

250 possible advantages of BMA. In particular for small datasets the effect of model uncertainty can

251 be substantial - this is disrespected in the framework of traditional regression modelling. 
252 Variable selection is often employed, however, when it is non-blinded to the outcome, it leads to

253 models that are biased in virtually all of their parameters. For small sample sizes, the sound

254 alternatives - such as regularization - might lead to results that are clinically not meaningful.

255 BMA is a relevant alternative, which avoids these issues by explicitly considering many models.

256 Our study has considerable limitations. The observational nature and low patient number

257 being probably the most important ones. The number of excluded patients with significant

258 alcohol intake has also to be dealt with caution. Since alcohol consumption was assessed by self-

259 reporting, there is a possibility that not all affected individuals were identified. Moreover, the

260 distance between the HIV center, where screening occurred and the hepatology center where

261 transient elastography measurement took place was the main reason potential participants

262 refused participation in the study. This could lead to selection bias, since low-compliance

263 patients could be underrepresented in the study population.

\section{Conclusions}

In conclusion, using previously described cutoff values we identified a high prevalence of hepatic fibrosis in HIV mono-infected patients. Our findings shed light on the relevance of HIVinduced immune dysregulation and overweight in the ageing HIV-infected population. The negative association between LS and the presence of lipodystrophy may reflect on the protective effect of prolonged exposure to antiretroviral therapy.

Fueled by the ongoing silent epidemic of obesity, the burden of liver diseases in individuals living with HIV shifts away from viral hepatitis coinfections to the NAFLD spectrum. A better understanding of factors leading to fibrosis will be the cornerstone of 
274 reduction in liver-related disease burden in the HIV-infected population. Nonetheless, further

275 controlled studies are warranted to clarify causal relations.

276

\section{Acknowledgements}

278

279

280

281

282

283

284

285

286

287

288

289

290

291

292

293

294

295

296

297

298

299

300

301

302

303

304

305

306

307
We are indebted to Erzsebet Varga, Kornelia Barbai, and Agnes Kissne Halasz for data collection and organization. We are also immensely grateful to Fiona O'Rourke for language correction.

\section{References}

Akhtar MA, Mathieson K, Arey B, Post J, Prevette R, Hillier A, Patel P, Ram L, Van Thiel DH, and Nadir A. 2008. Hepatic histopathology and clinical characteristics associated with antiretroviral therapy in HIV patients without viral hepatitis. Eur J Gastroenterol Hepatol 20:1194-1204. 10.1097/MEG.0b013e328305b9e0

Antiretroviral Therapy Cohort Collaboration. 2010. Causes of death in HIV-1-infected patients treated with antiretroviral therapy, 1996-2006: collaborative analysis of 13 HIV cohort studies. Clin Infect Dis 50:1387-1396. 10.1086/652283

Audsley J, Robson C, Aitchison S, Matthews GV, Iser D, Sasadeusz J, and Lewin SR. 2016. Liver Fibrosis Regression Measured by Transient Elastography in Human Immunodeficiency Virus (HIV)Hepatitis B Virus (HBV)-Coinfected Individuals on Long-Term HBV-Active Combination Antiretroviral Therapy. Open Forum Infect Dis 3:ofw035. 10.1093/ofid/ofw035

Blanco F, Barreiro P, Ryan P, Vispo E, Martín-Carbonero L, Tuma P, Labarga P, Medrano J, GonzálezLahoz J, and Soriano V. 2011. Risk factors for advanced liver fibrosis in HIV-infected individuals: role of antiretroviral drugs and insulin resistance. J Viral Hepat 18:11-16. 10.1111/j.13652893.2009.01261.x

Brunet L, Moodie EE, Young J, Cox J, Hull M, Cooper C, Walmsley S, Martel-Laferrière V, Rachlis A, Klein $\mathrm{MB}$, and Study CC-iC. 2016. Progression of Liver Fibrosis and Modern Combination Antiretroviral Therapy Regimens in HIV-Hepatitis C-Coinfected Persons. Clin Infect Dis 62:242-249. $10.1093 /$ cid/civ838

Chan AW, Patel YA, and Choi S. 2016. Aging of the Liver: What This Means for Patients with HIV. Curr HIV/AIDS Rep. 10.1007/s11904-016-0332-x

Costiniuk CT, Brunet L, Rollet-Kurhajec KC, Cooper CL, Walmsley SL, Gill MJ, Martel-Laferriere V, and Klein MB. 2016. Tobacco Smoking Is Not Associated With Accelerated Liver Disease in Human 
Immunodeficiency Virus-Hepatitis C Coinfection: A Longitudinal Cohort Analysis. Open Forum Infect Dis 3:ofw050. 10.1093/ofid/ofw050

DallaPiazza M, Amorosa VK, Localio R, Kostman JR, and Lo Re V. 2010. Prevalence and risk factors for significant liver fibrosis among HIV-monoinfected patients. BMC Infect Dis 10:116. 10.1186/1471-2334-10-116

Fernández-Montero JV, Barreiro P, Vispo E, Labarga P, Sánchez-Parra C, and Soriano V. 2013. Liver stiffness predicts liver-related complications and mortality in HIV patients with chronic hepatitis C on antiretroviral therapy. AIDS 27:1129-1134. 10.1097/QAD.0b013e32835e063f

Feuth T, van Baarle D, van Erpecum KJ, Siersema PD, Hoepelman Al, and Arends JE. 2014. CD4/CD8 ratio is a promising candidate for non-invasive measurement of liver fibrosis in chronic HCVmonoinfected patients. Eur J Clin Microbiol Infect Dis 33:1113-1117. 10.1007/s10096-014-20537

Gonzalez FA, Van den Eynde E, Perez-Hoyos S, Navarro J, Curran A, Burgos J, Falcó V, Ocaña I, Ribera E, and Crespo M. 2015. Liver stiffness and aspartate aminotransferase levels predict the risk for liver fibrosis progression in hepatitis C virus/HIV-coinfected patients. HIV Med 16:211-218. 10.1111/hiv.12197

González Guilabert MI, Hinojosa Mena-Bernal C, del Pozo González J, and del Pozo Pérez MA. 2010. [Retrospective study of FibroScan, APRI, FIB-4 and FORNS indexes compared with liver biopsy in the evaluation of liver fibrosis in patients with chronic hepatitis $\mathrm{C}$ monoinfection and HIV coinfection]. Gastroenterol Hepatol 33:425-432. 10.1016/j.gastrohep.2010.02.005

Grinspoon S, and Carr A. 2005. Cardiovascular risk and body-fat abnormalities in HIV-infected adults. $N$ Engl J Med 352:48-62. 10.1056/NEJMra041811

Han SH, Kim SU, Kim CO, Jeong SJ, Park JY, Choi JY, Kim do Y, Ahn SH, Song YG, Han KH, and Kim JM. 2013. Abnormal liver stiffness assessed using transient elastography (Fibroscan(R)) in HIVinfected patients without $\mathrm{HBV} / \mathrm{HCV}$ coinfection receiving combined antiretroviral treatment. PLoS One 8:e52720. 10.1371/journal.pone.0052720

Harrell FE. 2016. rms: Regression Modeling Strategies. R package version 4.5-0. https://CRAN.Rproject.org/package $=$ rms.

Hoeting JA, Madigan D, Raftery AE, and Volinsky CT. 1999. Bayesian model averaging: a tutorial. Statistical science:382-401.

Ioannou GN, Bryson CL, Weiss NS, and Boyko EJ. 2015. Associations between lipodystrophy or antiretroviral medications and cirrhosis in patients with HIV infection or HIV/HCV coinfection. Eur J Gastroenterol Hepatol 27:577-584. 10.1097/MEG.0000000000000290

Kliemann DA, Wolff FH, Tovo CV, Alencastro PR, Ikeda ML, Brandão AB, Barcellos N, and Fuchs SC. 2016. Biochemical non-invasive assessment of liver fibrosis cannot replace biopsy in HIV-HCV coinfected patients. Ann Hepatol 15:27-32.

Konerman MA, Mehta SH, Sutcliffe CG, Vu T, Higgins Y, Torbenson MS, Moore RD, Thomas DL, and Sulkowski MS. 2014. Fibrosis progression in human immunodeficiency virus/hepatitis $C$ virus coinfected adults: prospective analysis of 435 liver biopsy pairs. Hepatology 59:767-775. 10.1002/hep.26741

Kooij KW, Wit FW, van Zoest RA, Schouten J, Kootstra NA, van Vugt M, Prins M, Reiss P, van der Valk M, and Group ACS. 2016. Liver fibrosis in HIV-infected individuals on long-term antiretroviral therapy: associated with immune activation, immunodeficiency and prior use of didanosine. AIDS 30:1771-1780. 10.1097/QAD.0000000000001119

Li Vecchi V, Giannitrapani L, Di Carlo P, Mazzola G, Colletti P, La Spada E, Vizzini G, Montalto G, and Soresi M. 2013. Non-invasive assessment of liver steatosis and fibrosis in HIV/HCV- and HCVinfected patients. Ann Hepatol 12:740-748. 
400

401

Lui G, Wong VW, Wong GL, Chu WC, Wong CK, Yung IM, Wong RY, Yeung SL, Yeung DK, Cheung CS, Chan HY, Chan HL, and Lee N. 2016. Liver fibrosis and fatty liver in Asian HIV-infected patients. Aliment Pharmacol Ther. 10.1111/apt.13702

Macías J, Camacho A, Von Wichmann MA, López-Cortés LF, Ortega E, Tural C, Ríos MJ, Merino D, Téllez F, Márquez M, Mancebo M, and Pineda JA. 2013a. Liver stiffness measurement versus liver biopsy to predict survival and decompensations of cirrhosis among HIV/hepatitis C viruscoinfected patients. AIDS 27:2541-2549. 10.1097/QAD.0b013e32836381f3

Macías J, González J, Tural C, Ortega-González E, Pulido F, Rubio R, Cifuentes C, Díaz-Menéndez M, Jou A, Rubio P, Burgos A, and Pineda JA. 2014. Prevalence and factors associated with liver steatosis as measured by transient elastography with controlled attenuation parameter in HIV-infected patients. AIDS 28:1279-1287. 10.1097/QAD.0000000000000248

Macías J, Márquez $M$, Téllez $F$, Merino $D$, Jiménez-Aguilar $P$, López-Cortés LF, Ortega $E$, von Wichmann MA, Rivero A, Mancebo M, Santos J, Pérez-Pérez M, Suárez-Lozano I, Romero-Palacios A, TorresCornejo A, and Pineda JA. 2013b. Risk of liver decompensation among HIV/hepatitis C viruscoinfected individuals with advanced fibrosis: implications for the timing of therapy. Clin Infect Dis 57:1401-1408. 10.1093/cid/cit537

Macías J, Real LM, Rivero-Juárez A, Merchante N, Camacho A, Neukam K, Rivero A, Mancebo M, and Pineda JA. 2016. Changes in liver steatosis evaluated by transient elastography with the controlled attenuation parameter in HIV-infected patients. HIV Med. 10.1111/hiv.12384

Merchante N, Pérez-Camacho I, Mira JA, Rivero A, Macías J, Camacho A, Gómez-Mateos J, García-Lázaro M, Torre-Cisneros J, Pineda JA, and Infecciosas GApeEdIHVdISAdE. 2010. Prevalence and risk factors for abnormal liver stiffness in HIV-infected patients without viral hepatitis coinfection: role of didanosine. Antivir Ther 15:753-763. 10.3851/IMP1612

Njei B, McCarty TR, Luk J, Ewelukwa O, Ditah I, and Lim JK. 2016. Use of Transient Elastography in Patients with HIV-HCV Co-infection: A Systematic Review and Meta-analysis. J Gastroenterol Hepatol. 10.1111/jgh.13337

R Core Team. 2016. R: A language and environment for statistical computing. R Foundation for Statistical Computing, Vienna, Austria. https://www.R-project.org/.

Raftery A, Hoeting J, Volinsky C, Painter I, and Ka YY. 2015. BMA: Bayesian Model Averaging. R package version 3.18.6. https://CRAN.R-project.org/package=BMA

Raftery AE. 1995. Bayesian model selection in social research. Sociological methodology:111-163.

Redd AD, Wendel SK, Grabowski MK, Ocama P, Kiggundu V, Bbosa F, Boaz I, Balagopal A, Reynolds SJ, Gray RH, Serwadda D, Kirk GD, Quinn TC, and Stabinski L. 2013. Liver stiffness is associated with monocyte activation in HIV-infected Ugandans without viral hepatitis. AIDS Res Hum Retroviruses 29:1026-1030. 10.1089/AID.2013.0004

Rivero-Juárez A, Camacho A, Merchante N, Pérez-Camacho I, Macias J, Ortiz-Garcia C, Cifuentes C, TorreCisneros J, Peña J, Pineda JA, Rivero A, and (SAEI) GpeedlhvrHdISAdEI. 2013. Incidence of liver damage of uncertain origin in HIV patients not co-infected with HCV/HBV. PLoS One 8:e68953. 10.1371/journal.pone.0068953

Rockstroh JK, Mohr R, Behrens G, and Spengler U. 2014. Liver fibrosis in HIV: which role does HIV itself, long-term drug toxicities and metabolic changes play? Curr Opin HIV AIDS 9:365-370. 10.1097/COH.0000000000000064

Sandrin L, Fourquet B, Hasquenoph JM, Yon S, Fournier C, Mal F, Christidis C, Ziol M, Poulet B, Kazemi F, Beaugrand $M$, and Palau R. 2003. Transient elastography: a new noninvasive method for assessment of hepatic fibrosis. Ultrasound Med Biol 29:1705-1713.

Sanmartín R, Tor J, Sanvisens A, López JJ, Jou A, Muga R, Ojanguren I, Barluenga E, Videla S, Planas R, Clotet B, and Tural C. 2014. Progression of liver fibrosis in HIV/hepatitis C virus-coinfected 
402

403

404

405

406

407

408

409

410

411

412

413

414

415

416

417

418

419

420

421

422

423

424

425

426

individuals on antiretroviral therapy with early stages of liver fibrosis at baseline. HIV Med 15:203-212. 10.1111/hiv.12105

Serrano-Villar S, Sainz T, Lee SA, Hunt PW, Sinclair E, Shacklett BL, Ferre AL, Hayes TL, Somsouk M, Hsue PY, Van Natta ML, Meinert CL, Lederman MM, Hatano H, Jain V, Huang Y, Hecht FM, Martin JN, McCune JM, Moreno S, and Deeks SG. 2014. HIV-infected individuals with low CD4/CD8 ratio despite effective antiretroviral therapy exhibit altered T cell subsets, heightened CD8+ T cell activation, and increased risk of non-AIDS morbidity and mortality. PLoS Pathog 10:e1004078. 10.1371/journal.ppat.1004078

Shur NF, Tan Y, Goubet S, Fisher M, Gilleece Y, and Verma S. 2016. Non-viral liver disease burden in HIVmonoinfected individuals: a longitudinal observational retrospective cohort study. AIDS Care:16. 10.1080/09540121.2016.1191603

Sulyok M, Makara M, Rupnik Z, Ferenci T, Újhelyi E, Kormos L, Gerlei Z, Szlávik J, Horváth G, and VályiNagy I. 2015. Hepatic steatosis in individuals living with HIV measured by controlled attenuation parameter: a cross-sectional study. Eur J Gastroenterol Hepatol 27:679-685. 10.1097/MEG.0000000000000339

Vergara S, Macías J, Rivero A, Gutiérrez-Valencia A, González-Serrano M, Merino D, Ríos MJ, GarcíaGarcía JA, Camacho A, López-Cortés L, Ruiz J, de la Torre J, Viciana P, Pineda JA, and SAEI GpeEdIHVdl. 2007. The use of transient elastometry for assessing liver fibrosis in patients with HIV and hepatitis C virus coinfection. Clin Infect Dis 45:969-974. 10.1086/521857

Weber R, Sabin CA, Friis-Møller N, Reiss P, El-Sadr WM, Kirk O, Dabis F, Law MG, Pradier C, De Wit S, Akerlund B, Calvo G, Monforte A, Rickenbach M, Ledergerber B, Phillips AN, and Lundgren JD. 2006. Liver-related deaths in persons infected with the human immunodeficiency virus: the D:A:D study. Arch Intern Med 166:1632-1641. 10.1001/archinte.166.15.1632 
Figure 1 (on next page)

Recruitment flow of the study participants 


\section{invited to participate}

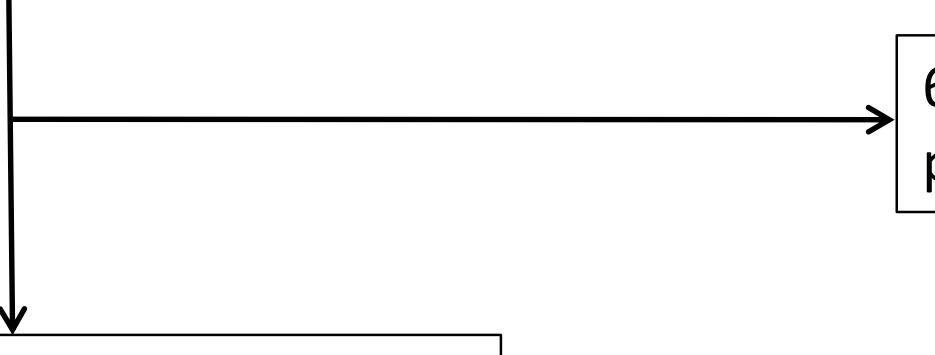

617 individuals did not show interest in participating

139 individuals provided informed consent

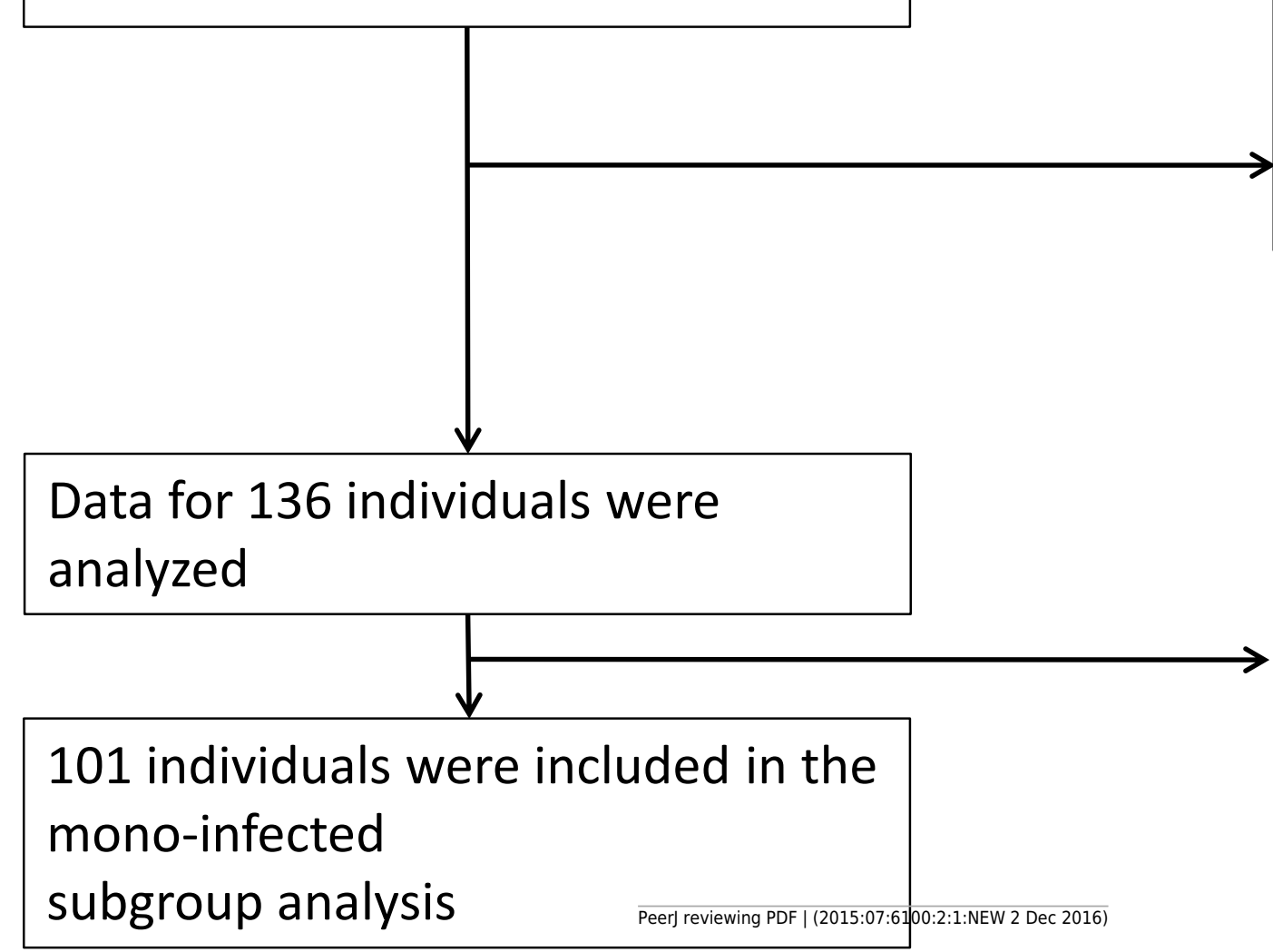

3 individuals were excluded :

- unreliable Fibroscan measurement $(n=3)$

- pregnancy $(n=0)$

35 individuals were excluded :

- anti-HCV positivity $(n=13)$

- anti-HBc or HBsAg positivity $(n=24)$

- alcohol intake $>50$ g daily $(n=4)$;

multiple criteria listed above were identified in 5 individuals 


\section{Figure 2 (on next page)}

Correlations between continuous variables and liver stiffness

The blue line shows the best-fitting linear curve, the red line shows the LOWESS-smoother.

Panels (A-I) refer to the correlation between liver stiffness value and the corresponding variable. 


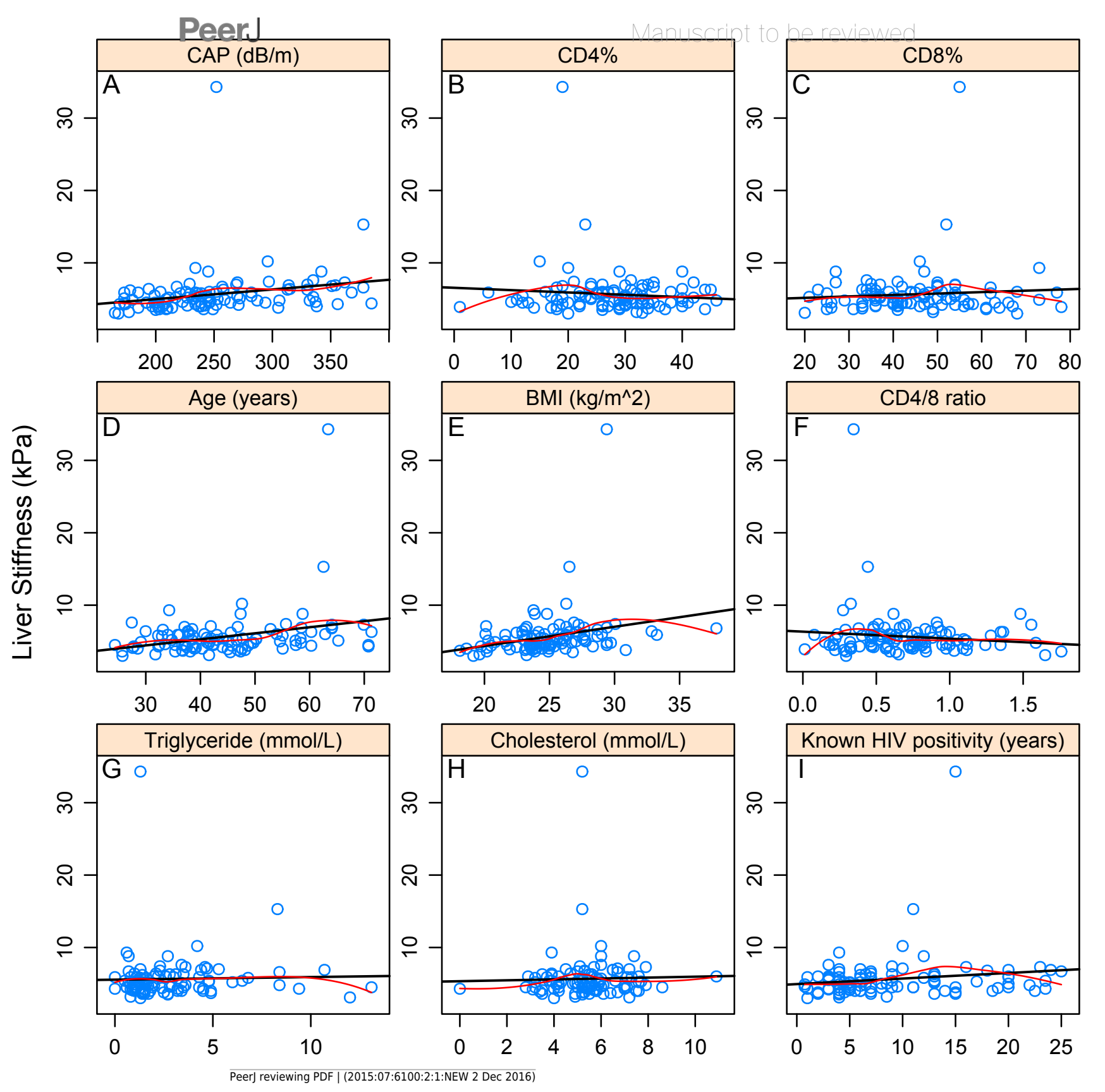


Figure 3 (on next page)

Models selected by BMA (Bayesian Model Averaging)

Red color displays negative, blue displays positive variable estimate (uncolored variables were not included in the model). On the $x$-axis, models are listed in the order of decreasing posterior model probability. 


\section{Table $\mathbf{1}$ (on next page)}

Study population $(n=101)$ characteristics

Due to missing values descriptive statistics of BMI (body mass index) and serum triglyceride values are derived from 100 individuals, serum cholesterol, the length of known HIV positivity and hypertension from 99 individuals. CAP: controlled attenuation parameter; ART: antiretroviral therapy 
1

Parameter

CD4 \%

$\mathrm{CD} 8 \%$

$\mathrm{CD} 4 / 8$ ratio

Age (years)

BMI (kg/m2)

Serum triglyceride $(\mathrm{mmol} / \mathrm{L})$

Serum cholesterol ( $\mathrm{mmol} / \mathrm{L})$

Known HIV positivity (years)

Liver Stiffness ( $\mathrm{kPa}$ )

$\mathrm{CAP}(\mathrm{dB} / \mathrm{m})$

ART ever taken

Darunavir

Atanazavir

Raltegravir

Etravirine

Nevirapine

Efavirenz

Tenofovir

Abacavir

Zidovudine

Lamivudine

Lopinavir

Gender (female)

Diabetes

Hypertension

Lipodystrophy
Mean (Median) \pm SD (IQR) [Min-Max]

27.6 (29) \pm 9 (11) [1-46]

45.1 (44) \pm 12.7 (17) [20-78]

$0.7(0.6) \pm 0.4(0.5)[0-1.8]$

$44.6(42.4) \pm 11.4$ (13.4) [24.4-71.3]

$25(24.8) \pm 3.2$ (3.3) [18.1-37.8]

$2.8(2) \pm 2.5(2.4)$ [0-13.1]

$5.4(5.4) \pm 1.5(1.8)$ [0-10.9]

$9.2(7) \pm 6.4$ (9) [0.8-25]

$5.7(5.1) \pm 3.3(1.7)$ [3-34.3]

$250.6(239) \pm 56.4$ (74) [165-385]

$\mathbf{N}(\%)$

$92(91.1)$

$20(19.8 \%)$

$7(6.9 \%)$

$8(7.9 \%)$

$9(8.9 \%)$

$22(21.8 \%)$

$27(26.7 \%)$

$38(37.6 \%)$

$13(12.9 \%)$

39 (38.6\%)

$89(88.1 \%)$

$26(25.7 \%)$

3 (3\%)

$11(10.9 \%)$

$21(21.2 \%)$

$12(11.9 \%)$ 


\section{Table 2 (on next page)}

Univariate analysis: associations between liver stiffness and continuous variables

The $p$-value pertains to the null hypothesis of no correlation; $p$-values are unadjusted; BMI: body mass index; CAP: controlled attenuation parameter 
1

Variable

CD4\%

$\mathrm{CD} 8 \%$

$\mathrm{CD} 4 / 8$ ratio

Age (years)

BMI $\left(\mathrm{kg} / \mathrm{m}^{2}\right)$

Triglyceride (mmol/L)

Cholesterol (mmol/L)

Known HIV positivity (years)

$\mathrm{CAP}(\mathrm{dB} / \mathrm{m})$

\section{Pearson}

$r$

$-0.087$

0.075447

$-0.10605$

0.285574

0.255489

0.026998

0.028166

0.147292

0.226115 $p$

0.386973

0.453335

0.291208

0.003794

0.010303

0.78975

0.781974

0.145703

0.022985

\section{Kendall}

$\tau$

$0.008555 \quad 0.901708$

$-0.01846 \quad 0.789103$

$-0.00341 \quad 0.960177$

$0.185478 \quad 0.006593$

$0.26108 \quad 0.000146$

$0.079497 \quad 0.250808$

$0.059661 \quad 0.3915$

$0.126008 \quad 0.073529$

$0.295207 \quad 0.0000162$

2 


\section{Table 3(on next page)}

Univariate analysis: associations between the liver stiffness and categorical variables

Liver stiffness (LS) values are presented in mean (median) $\pm \mathrm{SD}(\mathrm{IQR})$ [minimum-maximum] format. $p$-value pertains to the null hypothesis of stochastic equivalence of the two populations (presence/absence). ART: antiretroviral therapy 
1

Categorical
variable
ART ever taken
Darunavir
Atanazavir
Raltegravir
Etravirine
Nevirapine
Efavirenz
Tenofovir
Abacavir
Zidovudine
Lamivudine
Lopinavir
Gender (female)
Diabetes
Hypertension
Lipodystrophy

2
LS in the presence of variable LS in the absence of variable

$$
\begin{aligned}
& n=92,5.7(5.2) \pm 3.4(1.8)[3.1-34.3] \\
& n=20,5.6(5.3) \pm 1.7(2)[3.5-10.2] \\
& n=7,5.3(5.2) \pm 1.3(1.6)[3.6-7.3] \\
& n=8,6.2(5) \pm 3.7(0.7)[3.9-15.3] \\
& n=9,4.9(4.8) \pm 1(1.9)[3.6-6.3] \\
& n=22,5.3(5.3) \pm 1.1(1.8)[3.6-7.4] \\
& n=27,5.4(5.3) \pm 1.4(2.3)[3.1-8.8] \\
& n=38,6.3(5.3) \pm 5.1(2.1)[3.1-34.3] \\
& n=13,5.6(5.8) \pm 1.8(2)[3.6-10.2] \\
& n=39,5.5(4.9) \pm 2(1.7)[3.7-15.3] \\
& n=89,5.7(5.1) \pm 3.5(1.8)[3.1-34.3] \\
& n=26,6.7(5) \pm 6.1(1.8)[3.6-34.3] \\
& n=3,4.7(4.9) \pm 0.6(0.5)[4-5.1] \\
& n=11,7(6.3) \pm 3.3(2.6)[3.9-15.3] \\
& n=21,6.1(5.4) \pm 2.4(1.5)[4-15.3] \\
& n=12,5.3(5) \pm 1(1.6)[4-6.9]
\end{aligned}
$$

$$
\begin{array}{ll}
\mathrm{n}=9,4.9(4.3) \pm 1.9(1.9)[3-9.3] & 0.13281 \\
\mathrm{n}=81,5.7(5) \pm 3.6(1.7)[3-34.3] & 0.41051 \\
\mathrm{n}=94,5.7(5) \pm 3.4(1.7)[3-34.3] & 0.84091 \\
\mathrm{n}=93,5.6(5.2) \pm 3.3(1.9)[3-34.3] & 0.91481 \\
\mathrm{n}=92,5.7(5.1) \pm 3.5(1.8)[3-34.3] & 0.42414 \\
\mathrm{n}=79,5.8(5) \pm 3.7(1.7)[3-34.3] & 0.85302 \\
\mathrm{n}=74,5.8(5) \pm 3.8(1.7)[3-34.3] & 0.59088 \\
\mathrm{n}=63,5.3(5) \pm 1.3(1.7)[3-10.2] & 0.54861 \\
\mathrm{n}=88,5.7(5) \pm 3.5(1.8)[3-34.3] & 0.81937 \\
\mathrm{n}=62,5.8(5.2) \pm 4(1.9)[3-34.3] & 0.94157 \\
\mathrm{n}=12,5.1(4.4) \pm 1.8(1.9)[3-9.3] & 0.22107 \\
\mathrm{n}=75,5.3(5.1) \pm 1.4(1.7)[3-10.2] & 0.65209 \\
\mathrm{n}=98,5.7(5.2) \pm 3.4(1.9)[3-34.3] & 0.44681 \\
\mathrm{n}=90,5.5(5) \pm 3.3(1.6)[3-34.3] & 0.06365 \\
\mathrm{n}=78,5.5(4.9) \pm 3.6(1.8)[3-34.3] & 0.04548 \\
\mathrm{n}=89,5.7(5.1) \pm 3.5(1.7)[3-34.3] & 0.82133
\end{array}
$$




\section{Table 4 (on next page)}

Results of Bayesian Model Averaging (BMA)

PEP: Posterior effect probability, EV: expected value of the posterior distribution of the parameter, SD: standard deviation, CAP: Controlled attenuation parameter, ART: Antiretroviral therapy 
1

2

\begin{tabular}{|c|c|c|c|}
\hline Variables & PEP (\%) & EV & SD \\
\hline Intercept & 100.0 & -0.5095082 & 3.712 .817 \\
\hline CD4\% & 17.1 & -0.0130202 & 0.034393 \\
\hline CD8\% & 12.9 & 0.0061567 & 0.020026 \\
\hline Age (years) & 84.5 & 0.0827192 & 0.048982 \\
\hline BMI $\left(\mathrm{kg} / \mathrm{m}^{2}\right)$ & 49.3 & 0.1213562 & 0.147793 \\
\hline $\mathrm{CD} 4 / 8$ ratio & 44.2 & -0.9654844 & 1.276 .960 \\
\hline Triglyceride (mmol/L) & 1.6 & -0.0005448 & 0.018470 \\
\hline Cholesterol (mmol/L) & 1.6 & -0.0011508 & 0.030622 \\
\hline Sex & 2.2 & -0.0277561 & 0.342417 \\
\hline Diabetes & 3.3 & 0.0331575 & 0.282103 \\
\hline Hypertension & 1.5 & 0.0001101 & 0.106425 \\
\hline Lipodystrophy & 44.0 & -11.266 .415 & 1.508 .412 \\
\hline Known HIV positivity (years) & 2.4 & 0.0004474 & 0.015563 \\
\hline Darunavir & 2.0 & -0.0092738 & 0.147354 \\
\hline Atanazavir & 1.9 & -0.0139616 & 0.231754 \\
\hline Raltegravir & 1.5 & 0.0012294 & 0.162407 \\
\hline Etravirine & 8.5 & -0.1378151 & 0.583234 \\
\hline Nevirapine & 2.9 & -0.0197049 & 0.183840 \\
\hline Efavirenz & 2.2 & -0.0102161 & 0.134693 \\
\hline Tenofovir & 14.0 & 0.1519821 & 0.461496 \\
\hline Abacavir & 1.6 & 0.0022876 & 0.135247 \\
\hline Zidovudine & 10.5 & -0.1116331 & 0.406437 \\
\hline Lamivudine & 1.5 & 0.0027499 & 0.132358 \\
\hline Lopinavir & 26.7 & 0.3950730 & 0.778517 \\
\hline $\mathrm{CAP}(\mathrm{dB} / \mathrm{m})$ & 8.9 & 0.0009069 & 0.003627 \\
\hline
\end{tabular}

